Note

\title{
Plasma Sterilization of Polyethylene Terephthalate Bottles by Pulsed Corona Discharge at Atmospheric Pressure
}

\author{
SATOSHI MASAOKA* \\ Packaging Laboratory, Dai Nippon Printing Co., Ltd., 15-1, \\ Kamiya, 3-chome, Kita-ku, Tokyo 115-0043, Japan \\ Present address:Integrated Packaging Development Center, \\ Dai Nippon Printing Co., Ltd., 1-1-1, Ichigaya-Kagacho, \\ Shinjuku-ku, Tokyo 162-8001, Japan
}

Received 19 September, 2006/Accepted 22 January, 2007

\begin{abstract}
A pulsed power supply was used to generate a corona discharge on a polyethylene terephthalate bottle, to conduct plasma sterilization at atmospheric pressure. Before generating such a discharge, minute quantities of water were attached to the inner surface of the bottle and to the surface of a high voltage (HV) electrode inserted into the bottle. Next, highvoltage pulses of electricity were discharged between electrodes for $6.0 \mathrm{~s}$, while rotating the bottle. The resulting spore log reduction values of Bacillus subtilis and Aspergillus niger on the inner surface of the bottle were 5.5 and 6 or higher, respectively, and those on the HV electrode surface were each 6 or higher for both strains. The presence of the by-products gaseous ozone, hydrogen peroxide, and nitric ions resulting from the electrical discharge was confirmed.
\end{abstract}

Key words : Plasma sterilization/Pulsed discharge/Corona discharge/Atmospheric pressure/ PET bottle.

Chemical sterilization techniques using peroxides are used widely as means of sterilizing plastic packaging materials. However, in some cases, such chemical methods involve the problem of residues of chemicals used in sterilization on the materials. For this reason, our company has developed a low-cost system that can sterilize packages in-line and on a continuous basis, using no sterilizing agents. Such equipment is required to achieve spore log reduction $(S L R)$ values of 4 or higher in several seconds. For example, there have been cases in which the inner surfaces of polyethylene terephthalate (PET) bottles have been sterilized in-line in a continuous sterilization processing prior to filling the bottles with beverage.

Although irradiation such as electron beams or $\gamma$ rays have been put to practical use in sterilization techniques using no sterilizing agents, from the point

\footnotetext{
${ }^{*}$ Corresponding author. Tel: +81-3-3903-8135, Fax : +81-3-3903-8107.
}

of view of the costs of such equipment, such irradiation is not applicable to continuous, in-line sterilization.

Since roughly 1980 , there have been numerous reports made concerning plasma sterilization technologies. Viewed in terms of the pressure of the gases converted to plasma, these can be categorized into methods used under reduced pressure and those used under atmospheric pressure. In general, reduced-pressure processes are more complex and expensive than atmospheric pressure processes (Moisan et al., 2001). On the other hand, although there have been reports on glow discharge sterilization under atmospheric pressure using many kinds of power supplies, such as a radio frequency power supply (Rf) (Kelly-Wintenberg et al., 1998) and an alternating current (AC) supply (Moisan et al., 2001), since it is difficult to put large distances between electrodes using these power supplies it is not suited for sterilization of three-dimensional packages. Although the use of corona discharge using a pulsed 
power supply, which enables the establishment of large distances between electrodes, has been reported as a surface treatment for automotive bumpers (Totizawa, 1993), as far as is known by the author such a method has not been put to practical use as a sterilization method.

For these reasons, plasma sterilization was applied at atmospheric pressure, by generating corona discharge using a pulsed power supply, to PET bottles.

Before generating this discharge, minute quantities of water were attached as condensed water vapor to both the inner surface of the bottle and to the surface of a high voltage (HV) electrode inserted into the bottle. Next, high-voltage pulses of electricity were discharged between the HV electrode and a uniquely formed vessel with a ground electrode for 6.0 s, while rotating the bottle. This paper reports on the spore sterilization effects on Bacillus subtilis and Aspergillus niger and the by-products formed under these conditions, and proposes the sterilization mechanism. In addition, for practical use this paper discusses the fundamental technologies for developing a low-cost sterilization system that enables in-line continuous sterilization processing.

Fig. 1 shows a schematic of the equipment used to inject water vapor and mixed gases consisting of Ar, $\mathrm{O}_{2}$ and $\mathrm{N}_{2}$ simultaneously into the PET bottle. A metal pipe runs through a heating device and was heated by the heater to maintain a temperature of $180^{\circ} \mathrm{C}$. All piping beyond the heating device consisted of a stainless steel pipe, maintained at a temperature of $110^{\circ} \mathrm{C}$. Water and the mixed gas were run through this pipe to generate a mixed gas including water vapor. The bottle was placed on the outlet from the pipe, and $500 \mathrm{ml}$ of the mixed gas, containing $0.1 \mathrm{~g}$ of water vapor, was injected into the bottle by opening a solenoid valve. The condensation of some of the water vapor on the inner surface of the bottle resulted in attaching the water uniformly as a cloud on the sur-

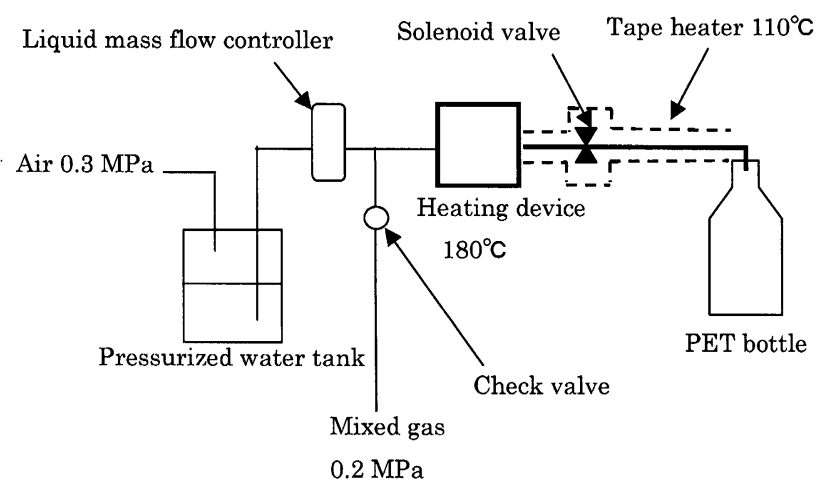

FIG. 1. Schematic of the apparatus for injecting mixed gas and water vapor simultaneously. face. Fig. 2 shows a schematic of the discharge apparatus. As a ground electrode at the bottom of the bottle, a dielectric substance was set on a rotating stand made of stainless steel, and the PET bottle with the mixed gas and water vapor injected was placed on the stand. Next, a HV electrode connected to the pulsed power supply was inserted into the bottle. A ground electrode on the side of the bottle was installed so that it covered one-half of the bottle. Fig. 3 shows a schematic of the side ground electrode vessel. This ground electrode features stainless steel mesh attached to the outside of an acrylic dielectric. The clearance between the acrylic dielectric and the bottle was $6 \mathrm{~mm}$, and the mesh was attached up to the same height as that of the bottle.

After B. subtilis ATCC 6633 and A. niger IAM 2020 were respectively plated and spoluration confirmed, spores were collected, washed, and suspended in sterilized water. Using an air atomizing spray, each spore suspension was sprayed inside the PET bottle

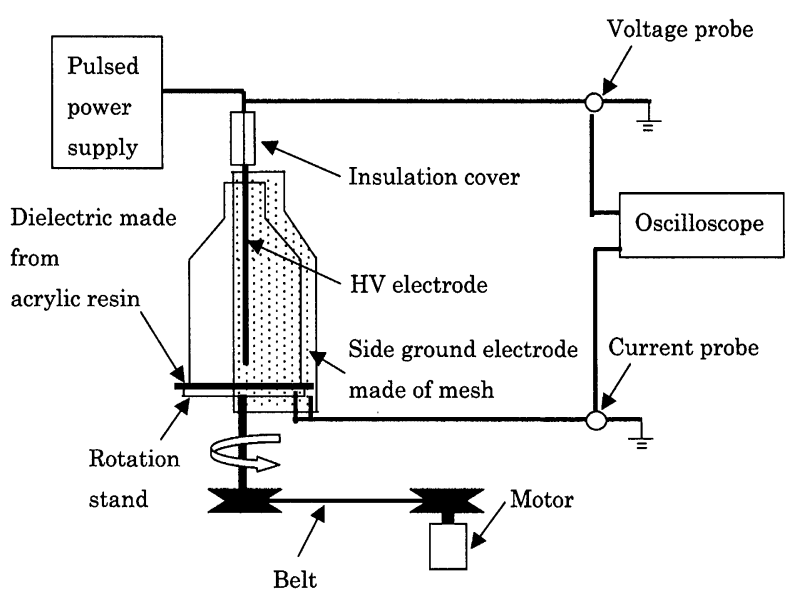

FIG. 2. Schematic of the discharge apparatus.

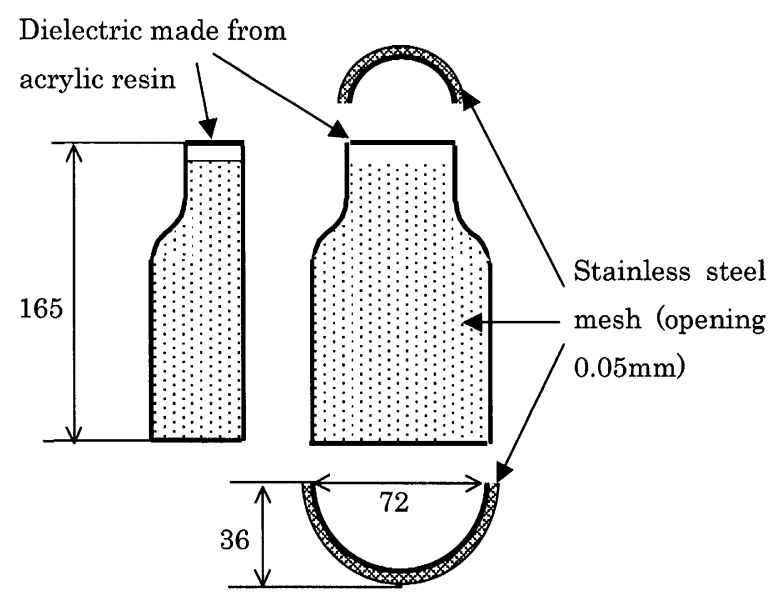

FIG. 3. Schematic of the vessel with a side ground electrode. 
uniformly, and a spontaneously dried bottle was used for evaluation of sterilization. The PET bottle used was a round one with a trunk diameter of $66 \mathrm{~mm}$, a height of $130 \mathrm{~mm}$, and a capacity of $250 \mathrm{ml}$, with $10^{4}$ or $10^{5}$ spores per bottle. Using a microsyringe, each spore suspension was inoculated through the insertion area to the bottle, in 10 spots at $10^{3}$ or $10^{4}$ spores per spot, and a spontaneously dried HV electrode used for evaluation of sterilization.

Using the apparatus shown in Fig. 1, the water vapor and the mixed gas were injected into the bottle to be used for evaluation of sterilization, and this bottle was immediately placed on the rotating stand shown in Fig. 2. Next, a HV electrode without spores was inserted into the bottle. A high-voltage pulse supply (8kW, Plasma Techno Research Inc., Tokyo) was then used for electrical discharge conducted over 6.0 s while rotating the bottle at $50 \mathrm{rpm}$. The peak voltage of the pulsed discharge was about $70 \mathrm{kV}$, the rise time was about $1.0 \mathrm{kV} / \mathrm{ns}$, the width was $90 \mathrm{~ns}$, the frequency was 1000 pulses per second, and the initial current discharged was approximately 105A. The bottle was collected immediately after receiving the discharge and $40 \mathrm{ml}$ of a Trypto-Soya Broth (Nissui Pharmaceutical Co., Ltd., Tokyo) was poured into the bottle, a presterilized cap was put on the bottle, and then the bottle was shaken sufficiently. The bottle with $B$. subtilis attached was incubated at $36^{\circ} \mathrm{C}$ for 14 $\mathrm{d}$, and the bottle with $A$. niger was incubated at 30 ${ }^{\circ} \mathrm{C}$ for $14 \mathrm{~d}$.

In testing the sterilization of the HV electrode surface, for the purpose of attaching the appropriate quantity of water, the temperature of HV electrode surface was adjusted before inserting it into the bottle. Temperature adjustment was conducted by blowing warm air across the area in which the HV electrode was inserted until its surface area reached a temperature of $38^{\circ} \mathrm{C}$, as confirmed by a radiation thermometer. The HV electrode was then promptly inserted into a spore-free bottle in which water vapor and the mixed gas had been injected, and electricity was discharged as described above. After the electrical discharge, the HV electrode was removed from the bottle and immersed in a test tube containing Trypto-Soya Broth, which was shaken sufficiently. Next, the electrode was removed, a sterilization cap attached, and incubation conducted as described above. After incubation for $14 d$, a visual check for the growth of microorganisms was conducted.

Promptly following the electrical discharge, the gaseous ozone concentration inside the bottle was measured. Also promptly following the electrical discharge, the bottle was filled with sterilized deionized water and incubated at $22{ }^{\circ} \mathrm{C}$ for $3 d$, then the concentrations of hydrogen peroxide $\left(\mathrm{H}_{2} \mathrm{O}_{2}\right)$, nitrite ions $\left(\mathrm{NO}_{2}^{-}\right)$, and nitrate ions $\left(\mathrm{NO}_{3}^{-}\right)$in the water were measured. Also following the electrical discharge, the inside of the bottle was rinsed by sterilized deionized water and then in the same way the concentrations of gaseous ozone, $\mathrm{H}_{2} \mathrm{O}_{2}, \mathrm{NO}_{2}^{-}$, and $\mathrm{NO}_{3}^{-}$in the water were measured. Rinsing was conducted by inserting a nozzle with an internal diameter of $9 \mathrm{~mm}$ into a reversed bottle to a $10 \mathrm{~mm}$ depth and injecting pressurized, sterilized deionized water from the nozzle for $3 \mathrm{~s}$ (500ml).

Gaseous ozone concentration was measured using an EG-2001R ozone monitor (Ebara Jitsugyo Co., Ltd., Tokyo, detection limit $0.01 \mathrm{ppm}) . \mathrm{H}_{2} \mathrm{O}_{2}$ concentration was measured using Oritector Model 5 (Central Kagaku Corp., Tokyo, with a detection limit of $0.01 \mathrm{ppm}$ ). $\mathrm{NO}_{2}^{-}$and $\mathrm{NO}_{3}^{-}$concentrations were measured by ion chromatography (IC) (DX-320, Dionex Corp., California, with a detection limit of $0.01 \mathrm{ppm}$ ). Analytical conditions of IC were as follows: column: Ion PAC AG12A+AS12A, mobile phase: $2.7 \mathrm{mM}-\mathrm{Na}_{2} \mathrm{CO}_{3} / 0.3 \mathrm{mM}-\mathrm{NaHCO}_{3}$, flow rate: $1.5 \mathrm{ml} /$ min, detector: electric conductivity.

Sterilization tests were carried out 10 times on the inner surface of each PET bottle and the HV electrode surface, and the SLR value determination was made by the equation (1), according to (Kawamura 1992):

$$
S L R=\log N_{0}-\log (\ln (n / r)) \cdots(1)
$$

where $N_{0}$ is the initial spore number per test sample, $\mathrm{n}$ is the number of test samples, and $\mathrm{r}$ is the number of samples negative for growth. When all samples showed no growth (i.e., $r=10$ ), it was considered that the SLR value was beyond the value which was cal-

TABLE 1. SLR value of the PET bottle inner-surface

\begin{tabular}{cccc}
\hline Strain & $\begin{array}{c}\text { initial spore number } \\
\text { / bottle }\end{array}$ & $r$ & SLR value $^{b}$ \\
\hline B. subtilis & $1.3 \times 10^{4}$ & 10 & $>5.1$ \\
& $1.5 \times 10^{5}$ & 6 & 5.5 \\
\hline A. niger & $1.0 \times 10^{4}$ & 10 & $>5.0$ \\
& $1.8 \times 10^{5}$ & 10 & $>6.2$ \\
\hline
\end{tabular}

${ }^{a}$ Treatment time : $6.0 \mathrm{~s}$.

${ }^{b} S L R$ value was calculated from the equation (1) as $n=10$.

TABLE 2. SLR value of the high voltage electrode surface ${ }^{a}$

\begin{tabular}{cccc}
\hline Strain & $\begin{array}{c}\text { initial spore number } \\
\text { / electrode }\end{array}$ & $r$ & SLR value $^{b}$ \\
\hline B. subtilis & $1.3 \times 10^{4}$ & 10 & $>5.1$ \\
& $1.1 \times 10^{5}$ & 10 & $>6.0$ \\
\hline A. niger & $1.0 \times 10^{4}$ & 10 & $>5.0$ \\
& $1.2 \times 10^{5}$ & 10 & $>6.1$ \\
\hline
\end{tabular}

${ }^{a}$ Treatment time : $6.0 \mathrm{~s}$.

${ }^{\circ} S L R$ value was calculated from the equation (1) as $n=10$. 
culated as $r=9$. Tables 1 and 2 show the results of calculating SLR values based on these tests. The SLR values of $B$. subtilis and $A$. niger on the inner surface of the bottle were 5.5 and 6 or higher, respectively, and the SLR values on the HV electrode were each 6 or higher.

Table 3 shows the concentrations of gaseous ozone, $\mathrm{H}_{2} \mathrm{O}_{2}, \mathrm{NO}_{2}^{-}$, and $\mathrm{NO}_{3}^{-}$before and after rinsing. Although the presence of these by-products could be confirmed prior to rinsing, following rinsing all concentrations except that of $\mathrm{NO}_{3}^{-}$were at or below detection limits. Analyses using HPLC, LCMS, GCMS, and FDMS (Field Desorption Mass Spectrometry) were conducted to check for the presence of other by-products. However, the presence of by-products other than the compounds described above could not be detected.

Directly following 6.0 s of electrical discharge, the temperature of the internal surface of the PET bottle was less than $60^{\circ} \mathrm{C}$. Hence, it is considered that heat effects on sterilization were minor in this study. During the electrical discharge, the entire interior of the bottle was exposed to dark blue-violet emissions, and the emission spectrum was determined. It was confirmed peaks at $315,336,358,381$ and $750 \mathrm{~nm}$, but no clear peak at $254 \mathrm{~nm}$. Hence, the effect of UV light can be also ignored.

It has been reported that the application of $3 \mathrm{mg} / \mathrm{I}$ of gaseous ozone, approximately $1530 \mathrm{ppm}\left(22^{\circ} \mathrm{C}\right.$, $95 \% \mathrm{RH}$ ) to $B$. subtilis (spores) resulted in a $D$ value of $7.5 \mathrm{~min}$ (Ishizaki, 1986). Although in this study $43 \mathrm{ppm}$ of gaseous ozone was detected after the electrical discharge, since 43ppm-gaseous ozone was in contact with $B$. subtilis spores only for 6.0 s at most, it is considered that ozone gas sterilization effects were minor. Furthermore, it was confirmed that the synergistic effect of sterilization at the residual concentrations of $\mathrm{H}_{2} \mathrm{O}_{2}$ and $\mathrm{NO}_{3}^{-}$could be ignored.

The author was unable to confirm any spore transformation due to electrical discharge by observation with a scanning electron microscope. Leakage of proteins and nucleic acids from the spore due to spore

TABLE 3. By-product concentration before and after rinsing

\begin{tabular}{lcc}
\hline By-product & before rinsing ppm & after rinsing ppm \\
\hline $\mathrm{Gaseous}^{2}$ ozone & & \\
$\mathrm{H}_{2} \mathrm{O}_{2}{ }^{a}$ & 43.0 & $\mathrm{ND}^{b}$ \\
$\mathrm{NO}_{2}^{-\theta}$ & 0.16 & $\mathrm{ND}$ \\
$\mathrm{NO}_{3}^{-a}$ & 0.01 & $\mathrm{ND}$ \\
\hline
\end{tabular}

${ }^{a}$ Detection limits were $0.01 \mathrm{ppm}$.

${ }^{b}$ below the detection limit. breakdown could also not be confirmed. Consequently, it was considered that no spore breakdown occurred.

Next, the role of active oxygen radicals, whose sterilization effects have been suggested in much of the literature, was also studied. It was confirmed that when $\mathrm{Ar}, \mathrm{O}_{2}$ and $\mathrm{H}_{2} \mathrm{O}$, respectively were not added to the interior of the bottle, sterilization effects decreased markedly. For these reasons, it is surmised that the sterilization effects are related to the presence of these substances. $\left(\mathrm{N}_{2}\right.$ was added to control by-production of gaseous ozone, and it was confirmed that $\mathrm{N}_{2}$ had almost no effect on sterilization.) Laroussi et al. (2004) have proposed that conducting barrier discharge in air under atmospheric pressure using an $\mathrm{AC}$ supply would generate $\mathrm{O}_{3}$ via atomic oxygen (O) and superoxide ions $\left(\mathrm{O}_{2}^{-}\right)$from $\mathrm{O}_{2}$, and would also generate $\mathrm{NO}_{1}^{-}, \mathrm{NO}_{2}^{-}, \mathrm{NO}_{3}^{-}$and $\mathrm{OH}$ radi$\mathrm{cal}(\mathrm{OH} \cdot)$ from the reaction of these active oxygen species with $\mathrm{N}_{2}$ and $\mathrm{H}_{2} \mathrm{O}$. Kelly-Wintenberg et al. (1998) have also suggested the sterilization effects of $\mathrm{O}_{2}^{-}$and $\mathrm{OH} \cdot$ when conducting glow discharge in air under atmospheric pressure, using a Rf supply. Each of these active oxygen radicals is known to have strong oxidation effects, and life times and diffusion lengths in the aqueous phase have been reported: for $\mathrm{O}_{2}^{-}, 5 \mathrm{~ms}, 100 \mu \mathrm{m}$ and for $\mathrm{OH} \cdot, 70 \mathrm{~ns}-200$ $\mu \mathrm{s}, 20 \mathrm{~nm}$ (Misonou et al., 1990). In light of the above knowledge, this experiment also suggests the production of active oxygen radicals such as $\mathrm{O}_{2}^{-}$and $\mathrm{OH} \cdot$. In particular, it is surmised that when a minute quantity of water is attached to the surface to be sterilized before discharge, $\mathrm{OH} \cdot$ with a short life time is generated on-site, resulting in strong and efficient sterilization effects.

The present author also examined physical properties of the bottle. In terms of practical use, no changes could be confirmed due to the discharge in the areas examined: the PET resin's molecular weight, the bottle's compressive strength, or the bottle's dimensions, or color. Furthermore, the author designed a basic continuous in-line aseptic filling system for PET bottles. A schematic diagram of this system is shown in Fig. 4. This system is constructed as a chambered structure that maintains an aseptic state throughout the system. The system is composed of a wheel for attaching water, a discharge wheel, and a rinsing wheel. Each wheel is used to carry out a series of operations while the bottle runs through the system. One particular characteristic of this method is the fact that the system enables control of secondary contamination of the bottle following sterilization, because it sterilizes the HV electrode and the inner surface of the bottle at the same time. 


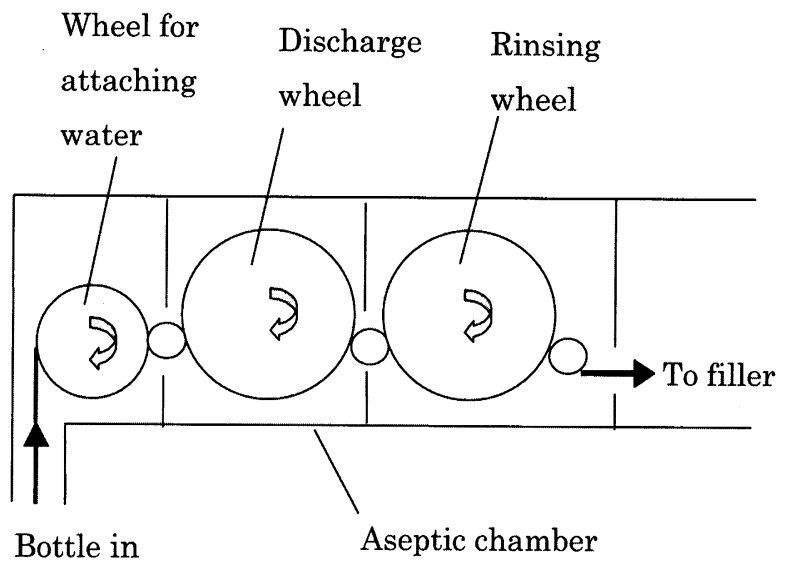

FIG. 4. Schematic diagram of a continuous in-line aseptic filling system for PET bottles.

Estimation of the equipment cost of such a system at low processing capabilities were roughly at or below the price of our company's sterilized filling system using $\mathrm{H}_{2} \mathrm{O}_{2}$, and running costs for electricity and gas were about one-half.

The high sterilization effects of this method was considered to result from attaching water directly to the surface to be sterilized and from the on-site generation of highly reactive $\mathrm{OH} \cdot$, and the low equipment cost was considered to result from the use of electrical discharge while rotating the bottle within a unique ground electrode.

With regard to future subjects of study, since it has been reported that Geobacillus stearothermophilus is a biological indicator for plasma sterilization using $\mathrm{H}_{2} \mathrm{O}_{2}$ (Jacobs, et al., 2001), there is a need to confirm sterilization effects on these bacteria spores as well. In addition, although the $\mathrm{NO}_{3}^{-}$concentration after rinsing in this experiment was very low at $0.04 \mathrm{ppm}$, since we are assuming sterilization using no sterilizing agents, there is a need for improvements with regard to $\mathrm{NO}_{3}^{-}$residue. In addition, at present the author is also studying the sterilization effects with largercapacity PET bottles such as those in $500-\mathrm{ml}$ size, and investing in high-speed systems with lower equipment costs (such as those capable of processing 600 bottles/min).

\section{REFERENCES}

Ishizaki. K., Shinriki, N., and Matsuyama, H. (1986) Inactivation of Bacillus spores by gaseous ozone, J. Applied Bacteriology, 60, 67-72.

Jacobs, P. T., and Lin, S-M. (2001) Sterilization process utilizing low-temperature plasma, In Disinfection, Sterilization and Preservation, Block, S.S (ed), Lippincott Williams \& Wilkins, Philadelphia.

Kawamura, I. (1992) Introduction to Validation, 2nd Edition (in Japanese), pp.42, Yakugyoujihou, Tokyo.

Kelly-Wintenberg, K., Montie, T. C., Brickman, C., Roth, jR. Carr, A. K., Sorge K., Wadsworth L. C., Tsai, P. P. Y. (1998) Room temperature sterilization of surfaces and fabrics with a one atmosphere uniform glow discharge plasma. J. Ind. Microbiol. Biotechnol., 20, 69-74.

Laroussi, M., and Leipold, F. (2004) Evaluation of the roles of reactive species, heat, and UV radiation in the inactivation of bacterial cells by air plasmas at atmospheric pressure. Int. J. Mass Spectrom., 233, 81-86.

Misonou, M., Osa, T., Niki, E., Morooka, Y. (1990) Chemistry of active oxygen species Kagaku Sosetsu 7 (in Japanese), pp10-17, Center for Academic Publications, Tokyo.

Moisan, M., Barbeau, J., Moreau, S., Pelletier, J., Tabrizian, M., and Yahia, L. H. (2001) Low-temperature sterilization using gas plasmas: a review of the experiments and an analysis of the inactivation mechanisms. Int. J. Pharm., 226, $1-21$.

Tochizawa, I. (1993) Atmospheric plasmas system for PP bumper coating. Techno-Cosmos, 4, 64-69. 